\title{
De la retórica a la acción: medir desigualdades en salud para "no dejar a nadie atrás"
}

\author{
Óscar J Mújica' y Claudia M Moreno²
}

Forma de citar Mújica OJ, Moreno CM. De la retórica a la acción: medir desigualdades en salud para "no dejar a nadie atrás". Rev Panam Salud Publica. 2019;43:e12. https://doi.org/10.26633/RPSP.2019.12

RESUMEN La equidad en salud es un principio rector de la acción en salud pública-cuyo noble propósito es construir sociedades más saludables y sostenibles y, al mismo tiempo, más justas e inclusivas. Ello se refleja en el compromiso mundial por 'no dejar a nadie atrás' que preside la Agenda 2030 para el desarrollo sostenible, aunque en ninguna de sus 169 metas se establezca ni conceptual ni cuantitativamente la reducción de desigualdades en salud. Reconociendo la urgencia de trascender la retórica y avanzar consecuentemente en la formulación y puesta en marcha de políticas sociales y de salud pro-equitativas -de lo local a lo global-, en este informe especial se revisan las bases conceptuales y metodológicas para el abordaje de la equidad en salud, se vinculan explícitamente en una propuesta instrumental y práctica que promueve el uso analítico de los datos administrativos disponibles desagregados subnacionalmente para informar la toma de decisiones en esa dirección, y se concluye planteando la necesidad de institucionalizar la medición, análisis y monitoreo de las desigualdades sociales en salud para crear efectivamente capacidades nacionales para actuar sobre los determinantes sociales y ambientales de la salud y rendir cuentas sobre el compromiso de no dejar a nadie atrás en el camino hacia el desarrollo sostenible, la salud universal y la justicia social.

Palabras clave Equidad en salud; disparidades en el estado de salud; determinantes sociales de la salud; teoría social; mediciones epidemiológicas.

La equidad en salud -el estado de ausencia de desigualdades injustas en las oportunidades para la salud de las personas y colectivos humanos debidas a circunstancias histórica o socialmente

\footnotetext{
Unidad de Análisis de Salud, Métricas y Evidencia, Departamento de Evidencia e Inteligencia para la Acción en Salud, Organización Panamerica de la Salud, Washington DC, Estados Unidos de América. La correspondencia se debe dirigir a Óscar Mújica. Correo electrónico: mujicaos@paho.org

2 Dirección de Epidemiología y Demografía, Ministerio de Salud y Protección Social, Bogotá DC, Colombia.
}

determinadas- es un valor social: una cualidad virtuosa aspiracional con protagonismo central en la toma de decisiones sociales, el conducto de la vida civilizada, la identidad de los pueblos y sus instituciones y la promoción de la salud poblacional (1). Y este valor social está adquiriendo alta visibilidad en las agendas políticas actuales, de lo local a lo global. De hecho, el compromiso de no dejar a nadie atrás que preside la Agenda 2030 para el Desarrollo Sostenible es, en su esencia, una invocación a la equidad social que incluye, desde luego, la equidad en salud (2).
Que la equidad se constituya hoy en el principio rector de la acción política en salud parece ser consecuencia de un doble imperativo contemporáneo, a juzgar por el peso de la evidencia que se viene acopiando: por un lado está el mandato moral -la inequidad ofende a nuestro sentido de justicia social- y, por otro, la exigencia política -la inequidad amenaza la gobernabilidad y la legitimidad democráticas (3-12). Construir y fortalecer capacidades institucionales para la rendición de cuentas sobre el principio de equidad en salud -ese no dejar a nadie atrás- se torna, por tanto, prioritario.

Este es un artículo de acceso abierto distribuido bajo los términos de la licencia Creative Commons Attribution-NonCommercial-NoDerivs 3.0 IGO, que permite su uso, distribución y reproducción en cualquier medio, siempre que el trabajo original se cite de la manera adecuada. No se permiten modificaciones a los artículos ni su uso comercial. Al reproducir un artículo no debe haber ningún indicio de que la OPS o el artículo avalan a una organización o un producto específico. El uso del logo de la OPS no está permitido. Esta leyenda debe conservarse, junto con la URL original del artículo. 
Esta alta y bienvenida visibilidad principista de la equidad en la Agenda de Desarrollo Sostenible reflejada, incluso, en uno de sus 17 objetivos (Objetivo 10: Reducir las desigualdades en los países y entre ellos), contrasta, no obstante, con la ausencia de enunciados ni conceptual ni cuantitativamente explícitos de reducción progresiva de desigualdades sociales en salud entre sus 169 metas y sus más de 230 indicadores (2).

En este informe especial se presentan los fundamentos conceptuales y metodológicos que guían la medición de desigualdades en salud y se propone que, desde un punto de vista práctico y operacional, medir de forma sistemática, es decir, monitorear las desigualdades sociales en la salud, tanto en el acceso a ella como en sus desenlaces, es un imperativo para avanzar con equidad hacia el desarrollo sostenible y generar credibilidad sobre el compromiso mundial de no dejar a nadie atrás.

\section{Bases conceptuales de la medición y el monitoreo de desigualdades en salud}

La medición de desigualdades en salud tiene su fundamento conceptual más explícito y su referente teórico más directo en el modelo de determinantes sociales de la salud propuesto por la Comisión sobre Determinantes Sociales de la Salud (CDSS) de la Organización Mundial de la Salud (OMS) en su Informe Final de 2008 (figura 1) (13). Dicho marco conceptual, a su vez, se basa e inspira en un conjunto de modelos análogos previos, notablemente los clásicos modelos de determinantes de la salud de Dahlgren y Whitehead (1991) (14) y de producción social de la salud de Diderichsen y Hallqvist (1998) (15), que destacan el papel determinante del contexto histórico-político y de la posición social en la generación, transmisión y perpetuación de desigualdades en la salud y el bienestar de las poblaciones (35). Más recientemente, la Comisión sobre Equidad y Desigualdades en Salud en las Américas ha publicado en 2018 un marco conceptual análogo más coherente con los Objetivos de Desarrollo Sostenible, en el cual se destacan los determinantes ambientales de la salud -incluido el cambio climático, el papel determinante de las estructuras culturales- y se subraya la etnicidad, el colonialismo persistente, el racismo estructural y el papel en la interseccionalidad y de los derechos humanos en las oportunidades para el disfrute colectivo de una vida digna (16-17).

De particular relevancia en el modelo conceptual de los determinantes sociales de la salud es la posición social, pues juega un papel explicativo fundamental. La posición social es un constructo que captura una cualidad ecológica cardinal de toda organización social: el gradiente social, esto es, la organización jerárquica de los miembros de una sociedad. La posición social se ve reflejada en diversas representaciones posibles, incluyendo el ingreso, la educación, la ocupación, el género, la pertenencia étnica y otras dimensiones proxy de, precisamente, los determinantes sociales. De acuerdo con el modelo conceptual vigente, la posición social determina la distribución de la salud y el bienestar en la población y tal determinación es mediada por los llamados determinantes proximales o intermediarios, que incluyen las circunstancias materiales, la cohesión social, la conducta humana, la herencia genética $\mathrm{y}$, de forma notable, la organización de los sistemas y los servicios de salud a todo nivel. Por su parte, la posición social misma es determinada por los denominados determinantes distales - que, parafraseando a Rose (18), Marmot identifica con acierto como "las causas de las causas" (13) - o determinantes estructurales, pues atañen directamente a las fuerzas y sistemas de la sociedad que determinan la distribución del poder, la riqueza, los recursos y el prestigio y que, en última instancia, hay que modificar positivamente para lograr la sociedad equitativa, inclusiva y saludable a la que todos aspiramos.

Alineada con el modelo de determinantes de la salud, la base axiológica de la medición de desigualdades en salud yace en el actual paradigma ecoepidemiológico (19-20), ese conjunto de modelos y valores que - reconociendo la presencia de múltiples niveles de organización (desde lo microcelular hasta lo macrosocial) y la compleja trama de interacción multicausal entre ellosforman una estructura coherente para entender y explicar la realidad observable y dotar de racionalidad a las políticas e intervenciones de salud pública que se proponen para mejorar esa realidad observada, superando el obsoleto paradigma del factor de riesgo, centrado exclusivamente en un único nivel: el

FIGURA 1. Modelo conceptual de los Determinantes Sociales de la Salud

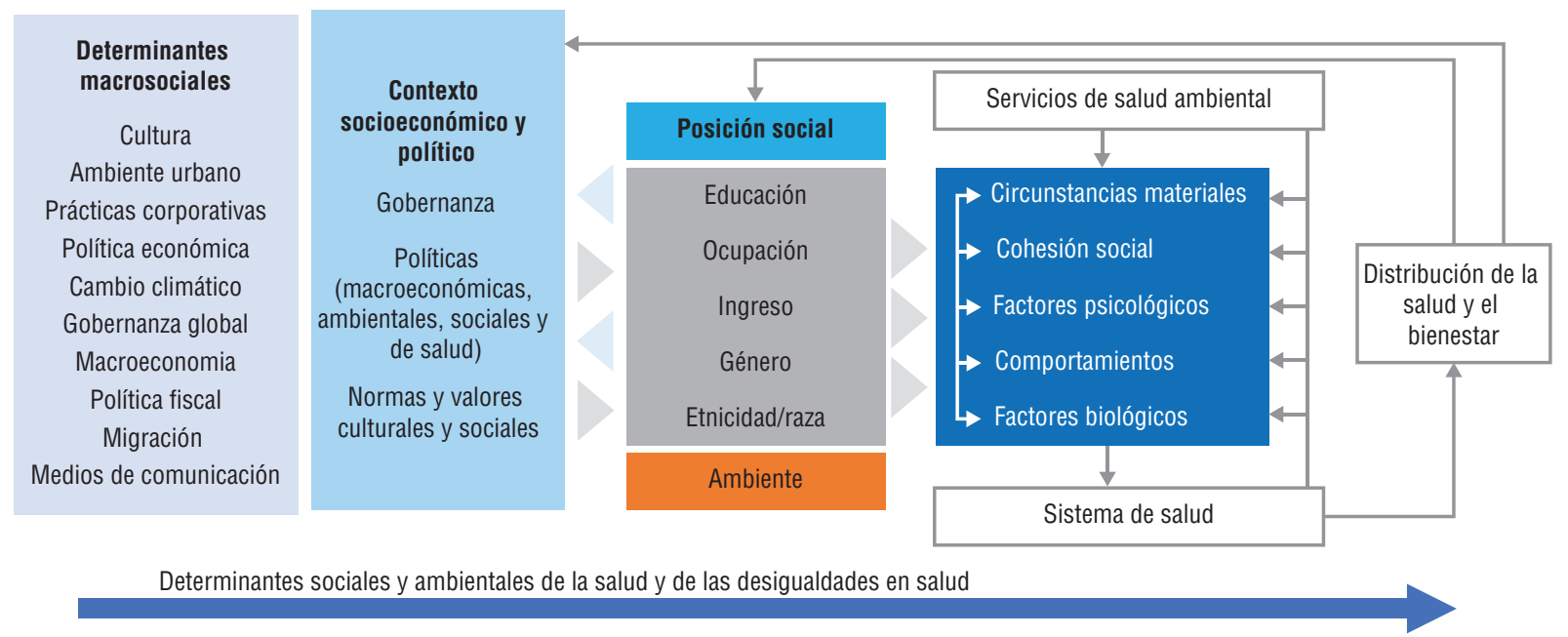

Fuente: modificado por los autores a partir de las referencias 13 y 35 . 
individuo (y muy frecuentemente, en un único atributo: la conducta humana, y, con ello, en una única intervención: el cambio de comportamiento). Una revisión más detallada de las cuestiones axiológicas y epistemológicas relativas a la medición de desigualdades en salud está descrita y disponible libremente en la bibliografía (21).

En la conceptualización de la medición de desigualdades en salud deberían tenerse en cuenta, al menos, otros cinco argumentos sustentatorios. Uno, los conceptos de "desigualdades en salud" y "determinantes sociales de la salud" están inextricablemente ligados, es decir, son inseparables. Como lo destaca el Informe Final de la CDSS, solamente es posible eliminar desigualdades en salud a través de la acción sobre los determinantes sociales de la salud $(13,16)$. Dicho a la inversa, el impacto poblacional de la acción -por antonomasia intersectorial- sobre los determinantes sociales de la salud solo puede medirse constatando una reducción no trivial de las desigualdades en salud.

Dos, en congruencia con el modelo de producción social de la salud y el marco conceptual de los determinantes sociales de la salud, las desigualdades en salud son consecuencia -efecto- de las desigualdades sociales; dicho de otro modo, las desigualdades en salud -las que importan para la salud pública- son desigualdades sociales en salud.

Tres, no es posible medir con rigor científico (es decir, con garantía de validez y reproducibilidad) las inequidades en salud; únicamente pueden medirse desigualdades en salud. Más concretamente, las desigualdades en salud se definen como diferencias oservables en la salud entre dos o más grupos socialmente determinados. Estas diferencias pueden objetivarse a partir de la observación simple e inambigua de datos relevantes (por ejemplo, la mortalidad infantil es más alta en niños pobres que en ricos). Las inequidades en salud, en cambio, no se miden, se juzgan. Más concretamente, las inequidades en salud se basan en un juicio ético sobre las desigualdades en salud observadas. Así, para determinar si una desigualdad observada constituye una inequidad en salud se debe juzgar como injusta, una valoración subjetiva que la identifica como arbitraria, innecesaria y evitable (por ejemplo, ¿es justo que la mortalidad infantil sea más alta en niños pobres que en niños ricos?) (22).
Un cuarto argumento, relacionado con el anterior, atañe a la imposibilidad intrínseca de preestablecer normativamente un estándar de oro o umbral predefinido sobre qué es inequitativo: corresponde a cada sociedad y momento histórico específicos definir qué desigualdad en salud constituye una afrenta a su sentido colectivo de justicia social y, por lo tanto, calificarla como una inequidad en salud. Tal elección de la sociedad está mediada por su particular nivel de aversión a la desigualdad, un importante atributo latente que también está históricamente determinado.

Por último, el quinto argumento que consideramos sustantivo presentar, es el de la acción: identificar una inequidad en salud (que, como se ha expuesto, es un acto de valoración moral) debe necesariamente verse acompañado -bajo el mismo imperativo moral- de la voluntad y decisión de intervenir sobre esa inequidad en salud y remediar la injusta situación. Denunciar una inequidad debe así generar incidencia política en pro de la equidad y la justicia social.

\section{Bases metodológicas de la medición y el monitoreo de desigualdades en salud}

Como en toda propuesta científica, el fundamento metodológico analítico de la medición de las desigualdades en salud es tributario del -y está supeditado almodelo conceptual: el marco de los determinantes sociales de la salud, que, como se ha mostrado, es un modelo causal multinivel cuya característica central es la posición social como determinante de la distribución de la salud y el bienestar en la población.

En su esencia, el enfoque metodológico básico encierra dos pasos simples: 1) la comparación del estado de salud de dos o más grupos poblacionales socialmente definidos y 2) la expresión de esa comparación en una métrica-resumen, específica y estándar, de la desigualdad en salud. Esta métrica-resumen puede expresar una brecha (la diferencia entre dos y solo dos grupos sociales, usualmente extremos) o bien un gradiente (la magnitud de la desigualdad en salud a lo largo de toda la jerarquía social), y puede ser absoluta (expresada en las mismas unidades de la variable del estado de salud) o bien relativa (sin dimensión, solo indicando la disproporcionalidad).
Desde un punto de vista más operacional, el enfoque metodológico puede ampliarse a tres pasos básicos a partir de un conjunto de datos disponibles. Tal conjunto puede consistir en un panel de datos ecológicamente agregados o puede ser una base de datos individuales. Estos datos tienen necesariamente que capturar tres dimensiones básicas: social, demográfica y de salud. La variable social, el proxy de determinante social, servirá para reproducir la jerarquía social en el análisis; la demográfica, para capturar el tamaño de los grupos de posición social (ponderación poblacional) y la de salud, para analizar su desigualdad distributiva en la población. Los tres pasos instrumentales anunciados son: 1) reproducir la jerarquía social, construyendo una escala de posición social relativa, 2) analizar la distribución de la salud sobre dicha escala de posición social, y 3) resumir el análisis de la distribución de la salud en ese gradiente social mediante el cálculo de métricas estándar de desigualdad en salud, según corresponda. Obsérvese que, al igual que el modelo conceptual, el modelo metodológico parte de la posición social: esta centralidad de la posición social para explicar y cuantificar la producción de desigualdades en la salud poblacional no se puede soslayar.

La figura 2 ilustra esquemáticamente estos tres elementos centrales en la conceptualización del enfoque analítico para medir desigualdades sociales en salud. Todo parte de la posición social: la jerarquía o gradiente social se construye usando la variable social disponible para ordenar el panel de datos desde la posición de mayor desventaja social hasta la de menor desventaja. Por ello, la variable social (el determinante social proxy) se lllama el estratificador de equidad. La Organización Panamericana de la Salud (OPS) ha propuesto un acrónimo para recordar la amplitud de posibles estratificadores de equidad: PROGRESAR, según se describe en la figura 2 (23). Con esto se cumple el primero de los tres pasos.

Para analizar la distribución de la salud sobre la escala de posición social así creada (el segundo paso), el analista tiene 3 opciones que no son, en absoluto, mutuamente excluyentes. La primera opción es optar por abreviar la distribución social mediante la partición por cuantiles (comunmente quintiles) y calcular la magnitud del indicador de salud (es decir, el promedio ponderado) para cada uno de estos grupos de 
FIGURA 2. Fundamento analítico para la medición de desigualdades en salud

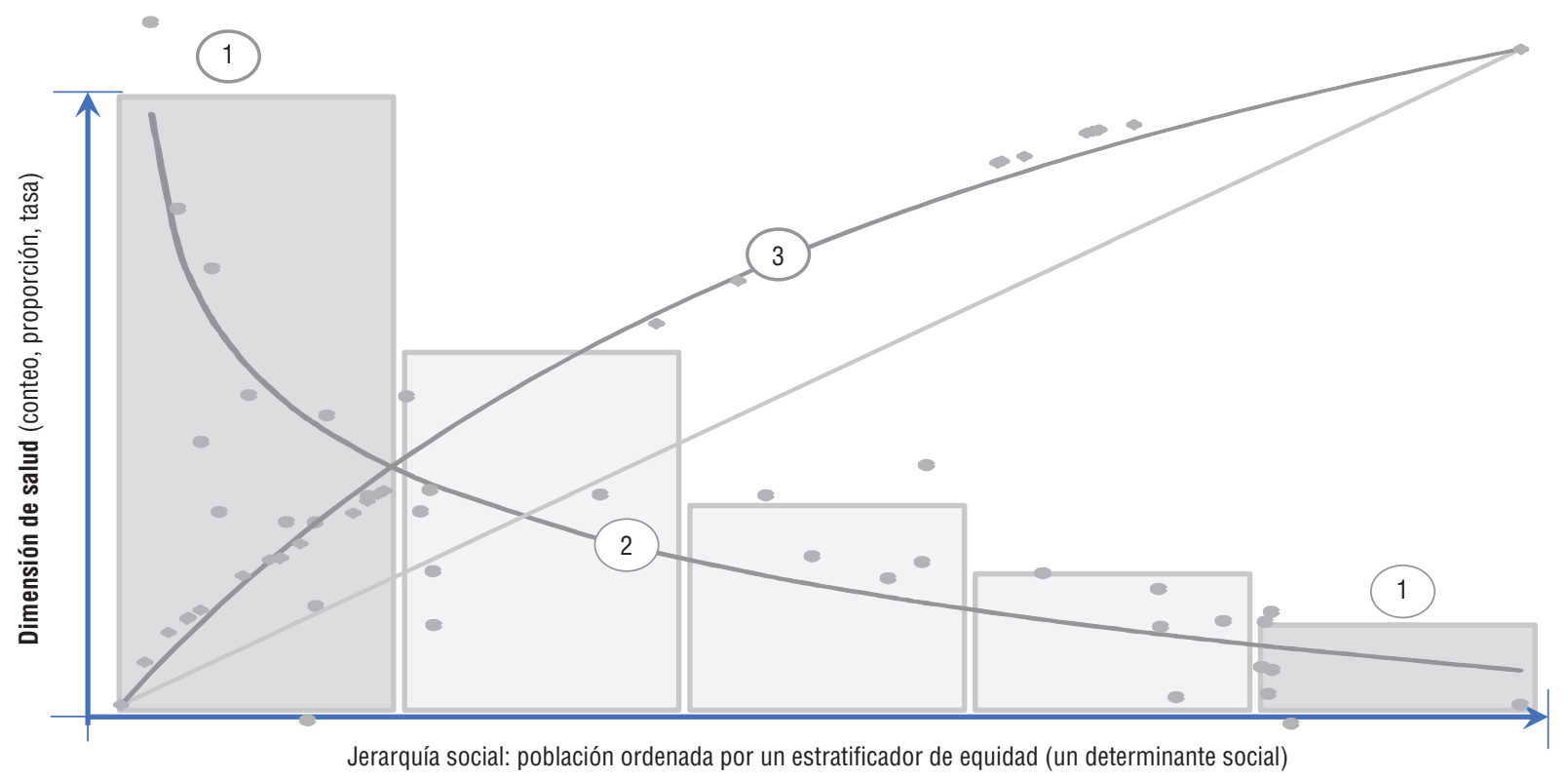

desde el más socialmente desaventajado
Estratificadores de Equidad: PROGRESAR

$\begin{array}{ll}\text { Plaza de residencia } & \text { Religión } \\ \text { Raza/etnicidad } & \text { Educación } \\ \text { Ocupación } & \text { Estado Socioeconómico } \\ \text { Género (sexo) } & \text { Ambiente y Recursos }\end{array}$

hasta el menos socialmente desaventajado
1. Partición por quintiles: índices de Kuznets absoluto y relativo (brecha absoluta y relativa)

2. Líneas de regresión:

3. Curvas de concentración: índice de desigualdad basado en la pendiente (gradiente absoluto)

índice de concentración de la desigualdad en salud (gradiente relativo)

Fuente: elaboración propia de los autores.

posición social así creados (los quintiles). Alternativamente, el analista podría adoptar la clasificación propia de variables sociales categóricas o nominales aquellas que no tienen un orden inherente preestablecido-, como, por ejemplo, los estratificadores de identidad: pertenencia étnica, género, orientación sexual, territorio, etc. Por otra parte, el analista puede optar por usar la distribución no-abreviada, en cuyo caso tiene las otras dos opciones de análisis de la distribución de la salud sobre el gradiente social: una es emplear técnicas de regresión estadística para modelizar el gradiente absoluto en salud, y la otra es construir una curva de concentración o de distribución de la disproporcionalidad entre la posición social y la carga de enfermedad (análoga a la curva de Lorenz, muy popular en econometría para estimar la desigualdad de ingreso con el coeficiente de Gini). Estas dos últimas opciones son metodológicamente más sofisticadas, pero también son más precisas, un elemento muy importante en el contexto del monitoreo.
El tercer y último paso -resumir la desigualdad en salud con una métrica estándar- se desprende del camino analítico seguido en el paso previo: si se trabaja con cuantiles, entonces se computan los índices de Kuznets: la brecha absoluta y la brecha relativa de desigualdad en salud, que no son más que la diferencia aritmética y el cociente o razón, respectivamente, de los valores del indicador de salud correspondientes a los quintiles extremos de la distribución social abreviada (sacrificando la información de los quintiles intermedios). Si se corre una regresión de las tasas de salud sobre la posición social, se computa el índice de desigualdad de la pendiente (IDP) o índice angular de desigualdad, que mide el grado de inclinación de la recta de regresión y, como tal, expresa en las mismas unidades de la variable de salud (como exceso de mortalidad o déficit de cobertura, por ejemplo) la magnitud de la desigualdad en salud asociada con la desigualdad social presente. Una regresión de este tipo - una técnica estadística muy poderosa- debiera ser capaz de capturar dos fenómenos ubícuos en el análisis de las desigualdades sociales en salud: uno es la heterocedasticidad o falta de homogeneidad de la varianza (es decir, varianza no constante), propia de la distinta talla poblacional de los grupos sociales; el otro es la asintoticidad o falta de linearidad de la relación entre la salud y lo social (especialmente observada con el ingreso), que refleja la ubicua ley de rendimientos decrecientes. Ello se resuelve con la inclusión de dos sofisticaciones metodológicas necesarias: una regresión por mínimos cuadrados ponderados y la transformación asintótica (logarítmica-exponencial) de los datos, según corresponda. En un contexto de monitoreo, el avance hacia la equidad se valora con la horizontalización de la curva de regresión (es decir, cuando el IDP tiende a cero). Si se trabaja con la curva de concentración, se computa el índice de concentración de la desigualdad en salud (ICS), que mide, en términos relativos (de $-1 \mathrm{a}+1)$, cuánto se concentra la carga de enfermedad en el extremo socialmente más desaventajado de la población (o viceversa). En un 
contexto de monitoreo, el avance hacia la equidad se valora con la diagonalización de la curva de concentración (es decir, cuando el ICS tiende a cero). En los dos últimos casos se rescata el principio de eficiencia estadística, que invita a utilizar todos los datos disponibles al computar una métrica resumen.

El enfoque analítico propuesto, con sus tres pasos instrumentales planteados, consiste en: 1) generar una escala de posición social relativa, 2) analizar la distribución de la salud en función de la posición social, y 3) resumir dicho análisis mediante el cálculo de métricas estándar de desigualdad en salud. Este enfoque se ha implementado en el Explorador de Equidad de la OPS (ExEq), una herramienta computacional basada en MS Excel (24) y el método descrito en detalle en una guía ilustrada paso a paso (25).

En la figura 3 se presenta un ejemplo -con datos reales- de la aplicación de estos conceptos para la medición de las desigualdades en la mortalidad infantil en el gradiente definido por el índice de necesidades básicas insatisfechas entre los 94 municipios del Departamento de Antioquia, en Colombia, que en 2015 registraron muertes de menores de un año. El análisis se basa en datos administrativos de dominio público compilados en el Sistema Integral de Información para la Protección Social (SISPRO) y extraídos por el Observatorio para Medición de Desigualdades y Análisis de Equidad en

FIGURA 3. Desigualdades en mortalidad infantil en el gradiente intermunicipal de satisfacción de necesidades básicas, Departamento de Antioquía, Colombia, 2015
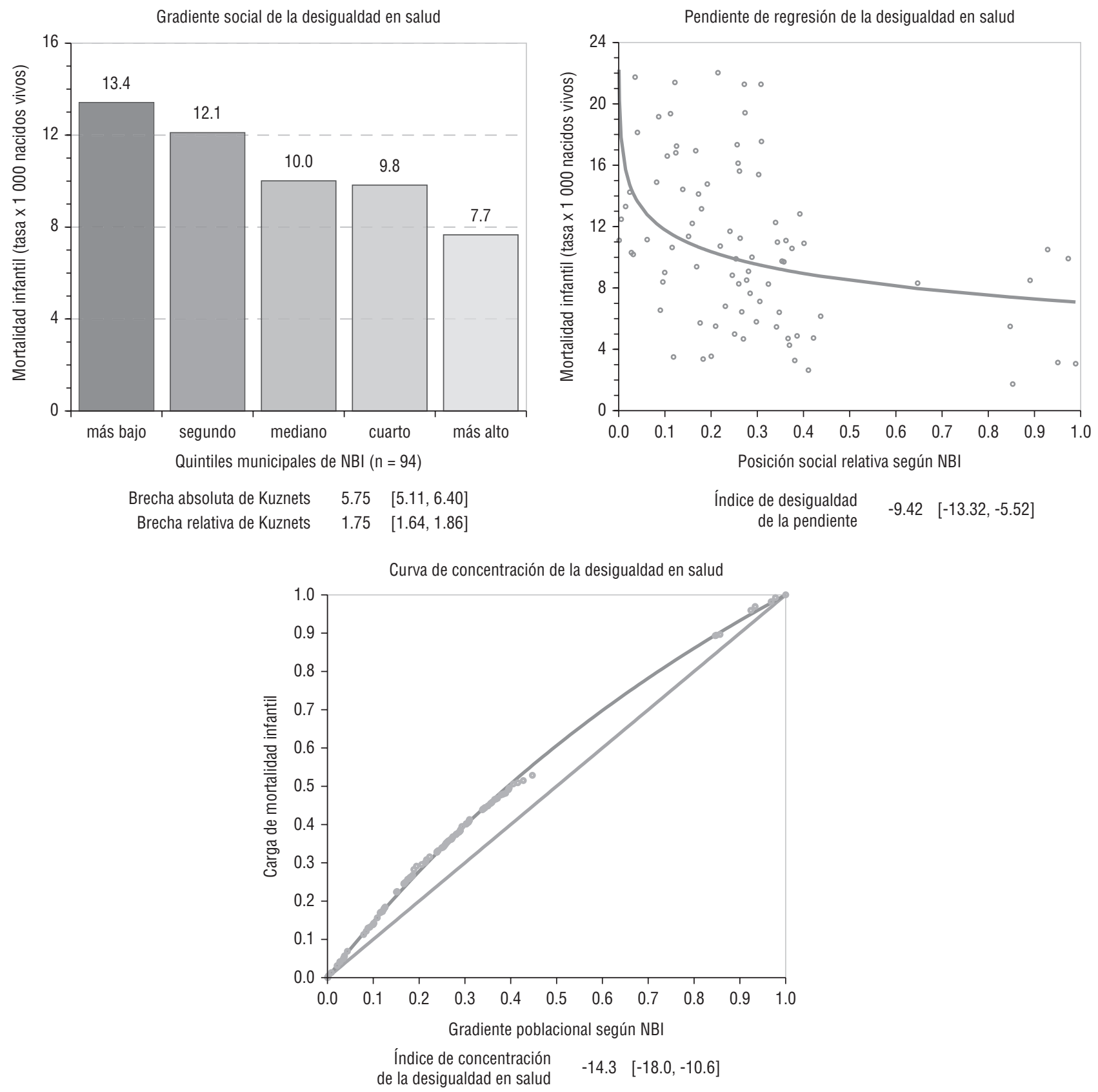

Nota: elaboración propia de los autores a partir de las referencias 26 y 24. 
Salud (ODES) del Ministerio de Salud y Protección Social de Colombia (26).

En el departamento de Antioquia, en 2015 (punto final de los Objetivos de Desarrollo del Milenio, ODM) se comprobó la presencia de notorias desigualdades en la supervivencia infantil (ODM 4) entre los 94 municipios según el nivel de insatisfacción de necesidades básicas (NBI). Entre los quintiles de municipios con más alto y más bajo NBI existe un exceso de mortalidad equivalente a casi 6 $(5,75)$ muertes infantiles por cada 1000 nacidos vivos (la brecha absoluta de desigualdad). El riesgo de muerte infantil en el quintil de municipios con más alto NBI es casi dos veces $(1,75)$ el riesgo del quintil de municipios con más bajo NBI (la brecha relativa de desigualdad). Si se tiene en cuenta todo el gradiente social por NBI (es decir, todos los municipios y no solamente los de los quintiles extremos) y, además, se considera el tamaño de la población de nacidos vivos de cada municipio, el análisis de regresión muestra un patrón de desigualdad congruente con exclusión marginal (del quintil con mayor NBI) y un exceso de mortalidad más intenso: casi $10 \quad(9,42)$ muertes infantiles por cada 1000 nacidos vivos (el gradiente absoluto de desigualdad). El índice de concentración (-14,3\%) señala la presencia de desigualdad negativa, es decir, una desproporcionada concentración de muertes infantiles en el extremo de municipios con mayor NBI. De hecho, el 20\% de población municipal de nacidos vivos con mayor NBI concentra alrededor de $30 \%$ del total de muertes infantiles del departamento ese año.

En la conceptualización del enfoque analítico para la medición de las desigualdades sociales en salud debe considerarse que estos tres pasos esenciales son necesarios y suficientes para generar la evidencia requerida para informar el proceso de formulación de políticas e intervenciones pro-equitativas en salud, así como para monitorear el impacto que, sobre la equidad, pueden tener $-\mathrm{o}$ dejar de tener- las intervenciones poblacionales sanitarias en curso. Aunque se reconoce que la medición de desigualdades sociales en salud es un proceso complejo y poco intuitivo y que, debido a ello, la creación, la consolidación y el sostenimiento de capacidades analíticas institucionales para su desarrollo en la generación de incidencia política en pro de la equidad es un proceso difícil, hay una creciente demanda social e igualmente un creciente compromiso institucional por mitigar efectivamente las profundas inequidades sociales en salud que infaustamente caracterizan a la Región de las Américas (12). Por otra

FIGURA 4. Patrones de desigualdad social en salud y estrategias de intervención. Tasa de mortalidad infantil por quintiles de ingreso. Encuestas DHS; países y años seleccionados en las Américas
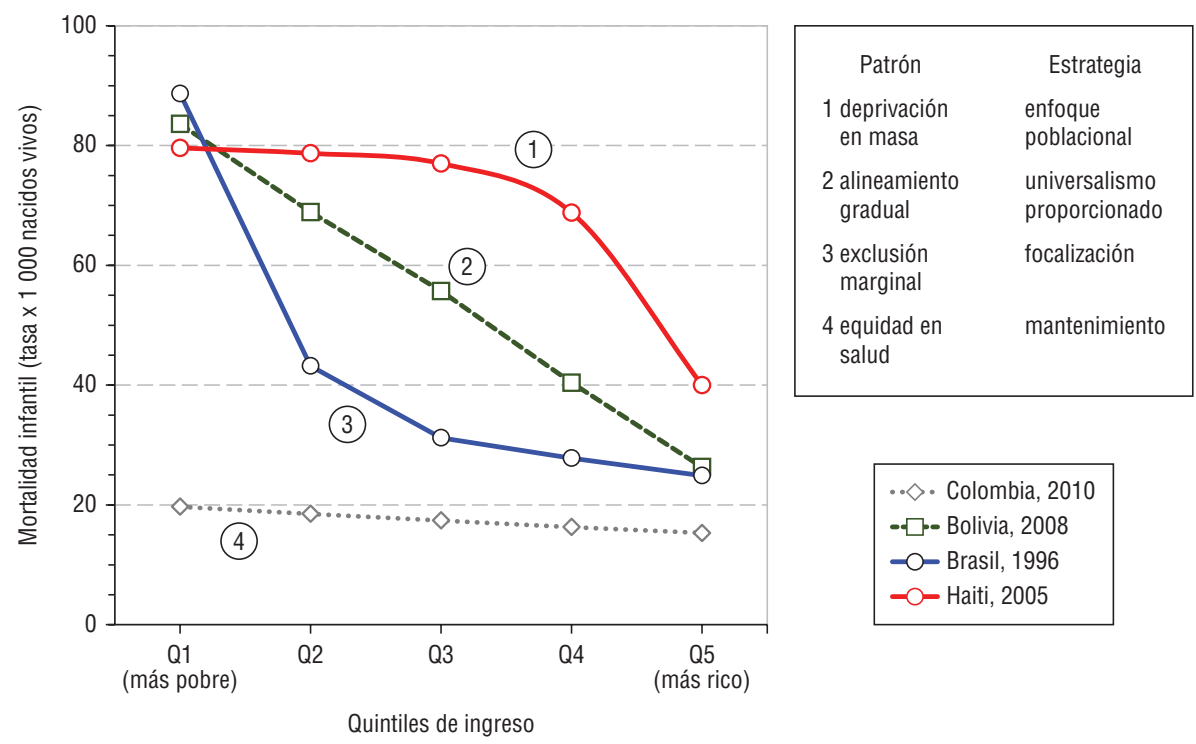

Fuente: elaboración propia de los autores a partir de la referencia 36. parte, ahora se comprueba una saludable convergencia conceptual, metodológica y hasta instrumental (gracias al poder computacional disponible) para la medición -y, en general, el abordajede las desigualdades en salud (23-25, 27-30) y se reconoce la subjetividad implícita en el proceso mismo de cuantificación de las desigualdades (31).

En este escenario contemporáneo no puede soslayarse la relevancia estratégica del principio de Tukey, que rescata la utilidad del análisis exploratorio de datos, especialmente de aquellos que provienen de registros administrativos (incompletos, imperfectos, inactuales): la intencionalidad analítica no debe ser reinvindicar hipótesis causales, sino extraer patrones ("pattern extraction, not causal claims") (32). La figura 4 ilustra con elegante elocuencia el principio de Tukey aplicado a la medición de desigualdades sociales en salud (36).

A partir de la aplicación de las bases conceptuales y metodológicas presentadas a un conjunto de datos relevantes es posible identificar y extraer patrones de desigualdad en salud (33); más aún, existe un correlato de intervenciones consistente y coherente con tales patrones, lo cual, a su vez, ilustra cómo el análisis puede informar la decisión política en salud (23-24, 34).

Como conclusión, la medición de desigualdades en salud tiene su fundamento axiológico en el modelo multinivel de determinantes sociales de la salud y el paradigma eco-epidemiológico. Es esencial reconocer la determinación social de las desigualdades en salud para defender la primacía de la equidad como valor social y como principio rector de la acción política en salud. Más relevante es aún este reconocimiento para generar capacidad de rendir cuentas sobre el compromiso por no dejar a nadie atrás en el camino hacia el desarrollo sostenible y la salud universal. Este compromiso demanda impostergablemente crear competencias profesionales y capacidades institucionales en la medición y el análisis de desigualdades sociales en salud, institucionalizar el monitoreo de las desigualdades en salud como ejercicio soberano de la primera función esencial de salud pública, es decir, el análisis de la situación de salud, y generar, tanto a nivel sectorial como intersectorial, incidencia política en pro de la equidad, como, por ejemplo, 
la modificación virtuosa de la asignación de recursos hacia la equidad y la justicia social.

Agradecimientos. Los autores expresan su reconocimiento a los organizadores del $10^{\circ}$ Congreso Internacional de Salud Pública Salud, Paz y Equidad Social (Universidad de Antioquia; Medellín, of population health. New York: Oxford University Press; 2018.

2. United Nations General Assembly. Transforming our World: the 2030 Agenda for Sustainable Development. A/ RES/70/1. New York: United Nations; 2015.

3. Bouchard L, Albertini M, Batista R, de Montigny J. Research on health inequalities: a bibliometric analysis (1966-2014). Soc Sci Med. 2015:141;100-8.

4. Göran T. The Killing Fields of Inequality. Cambridge: Polity Press; 2013.

5. Piketty T. Capital in the Twenty-First Century. Cambridge: The Bellknap Press of Harvard University Press; 2014.

6. Atkinson $\mathrm{AB}$. Inequality: what can be done? Cambridge: Harvard University Press; 2015.

7. Stiglitz JE. The Great Divide: unequal societies and what we can do about them. New York: W.W. Norton \& Company; 2015.

8. Deaton A. The Great Escape: health, wealth, and the origins of inequality. Princeton: Princeton University Press; 2013.

9. Marmot M. The Health Gap: the challenge of an unequal world. London: Bloomsbury; 2015.

10. Etienne CF. Achieving Social Equity. In: Frenk J, Hoffman SI, eds. "To Save Humanity": what matters most for a healthy future. New York: Oxford University Press; 2015.

11. Becerra-Posada F. Equidad en salud: mandato esencial para el desarrollo sostenible. Pan Am J Public Health. 2015;38(1):1-4.

12. Organización Panamericana de la Salud. Las Desigualdades Sociales en Salud. En: Salud en las Américas 2017: Panorama regional. Washington DC: Organización Panamericana de la Salud; 2017.

13. World Health Organization, Commission on Social Determinants of Health. Closing the gap in a generation: health equity through action on the social determinants of health. Final Report. Geneva: World Health Organization; 2008.

14. Dahlgren G, Whitehead M. Policies and Strategies to Promote Social Equity in Health. Stockholm: Institute for Futures Studies; 1991.
Colombia; octubre 2017), así como al Ministerio de Salud y Protección Social (MSPS) de la República de Colombia y al Equipo de País de la OPS/OMS por el constante apoyo a las acciones de cooperación técnica por la equidad en salud.

Conflictos de interés. Los autores declaran no tener conflictos de interés.

\section{REFERENCIAS}

15. Diderichsen F, Hallqvist J. Social Inequalities in Health: some methodological considerations for the study of social position and social context. En: Arve-Parès $\mathrm{B}$, ed. Inequality in Health-a Swedish perspective. Stockholm: Swedish Council for Social Research; 1998.

16. Organización Panamericana de la Salud. Sociedades justas, equidad en salud y vidas dignas. Resumen Ejecutivo del Informe Final de la Comisión OPS sobre Equidad y Desigualdades Sociales en Salud en las Américas. Washington DC: OPS; 2018

17. Marmot M. Just societies, health equity, and dignified lives: the PAHO Equity Commission. Lancet. 2018 Sep 24. pii: S0140-6736(18)32349-3. doi: 10.1016/ S0140-6736(18)32349-3.

18. Rose G. Sick individuals and sick populations. Int J Epidemiol. 1985;14(1):32-8.

19. Susser M, Susser E. Choosing a future for epidemiology: II. From black box to Chinese boxes and eco-epidemiology. Am J Public Health. 1996;85(5):674-7.

20. Krieger N. Epidemiology and the web of causation: has anyone seen the spider? Soc Sci Med. 1994; 39(7):887-903.

21. Mújica OJ. Cuatro cuestiones axiológicas de la epidemiología social para el monitoreo de la desigualdad en salud. Pan Am J Public Health 2015;38(6):433-41.

22. Whitehead M. The Concepts and Principles of Equity and Health. Copenhagen: World Health Organization; 1990.

23. Organización Panamericana de la Salud. Manual para el Monitoreo de las Desigualdades en Salud, con especial énfasis en países de ingresos medianos y bajos. Washington DC: Organización Panamericana de la Salud; 2016.

24. Pan American Health Organization. Equity Explorer: A health inequality pattern extractor; version 3. Washington DC: PAHO; 2017.

25. Organización Panamericana de la Salud. Guía ilustrada paso-a-paso para el cálculo de métricas estándar de desigualdad social en salud con uso del Explorador de Equidad. Washington DC: OPS, 2019. [En prensa.]

26. Ministerio de Salud y Protección Social. Observatorio para Medición de Desigualdades y Análisis de Equidad en Salud (ODES-Colombia). Bogotá DC:
Financiación. Este estudio no ha recibido financiación.

Declaración. Las opiniones expresadas en este manuscrito son responsabilidad de los autores y no reflejan necesariamente los criterios ni la política de la RPSP / PAJPH y/o de la OPS y/o del MSPS.
Ministerio de Salud; 2015. Disponible en: https: / / www.minsalud.gov.co/salud/ Paginas / Sistema Integralde Informaci \%C3\%B3nSISPRO.aspx Acceso el 7 de octubre de 2017.

27. Wagstaff A, Paci P, van Doorslaer E. On the measurement of inequalities in health. Soc Sci Med. 1991;33(5):545-57.

28. Mackenback JP, Kunst AE. Measuring the magnitude of socioeconomic inequalities in health: an overview of available measures illustrated with examples from Europe. Soc Sci Med. 1997; 44(6):757-71.

29. Kakwani N, Wagstaff A, van Doorslaer E. Socioeconomic inequalities in health: measurement, computation, and statistical inference. J Econometrics. 1997;77(1): 87-103.

30. Schneider MC, Castillo C, Bacallao J Loyola E, Mújica OJ, Vidaurre M, et al. Métodos de medición de las desigualdades de salud. Rev Panam Salud Publica. 2002;12(6):398-415.

31. Harper S, King NB, Meersman SC, Reichman ME, Breen N, Lynch J. Implicit value judgments in the measurement of health inequalities. The Milbank Quarterly. 2010;88(1):4-29.

32. Tukey JW. Exploratory Data Analysis Menlo Park: Addison-Wesley; 1977.

33. Becerra-Posada F, Mújica OJ. Equidad en salud para el desarrollo sostenible. Rev Salud Publica Nutr. 2016;15(1):16-26.

34. Mújica OJ. Trascendiendo la invisibilidad: el monitoreo de las desigualdades sociales en salud. Hacia Promoc Salud. 2016;21(1):9-11.

35. Galea S. Macrosocial determinants of population health. New York: Springer, 2007.

36. World Health Organization. Global Health Observatory's Health Equity Monitor. Geneva: WHO; 2014. Disponible en: https://www.who.int/gho/health_equity/ en/ Acceso el 15 de abril de2018.

Manuscrito recibido el 25 de mayo de 2018. Aceptado para publicación, tras revisión, el 11 de octubre de 2018. 
ABSTRACT Health equity is a guiding principle for public health action. Its noble purpose is to build healthier, sustainable societies that are also more just and inclusive. This is reflected in the global commitment to "leave no one behind", expressed in the 2030 Agenda for

From words to action: measuring health inequalities to "leave no one behind"

Keywords
Sustainable Development, although none of the Agenda's 169 targets focuses on reducing health inequalities, either conceptually or quantitatively. Recognizing the urgency to go beyond words and move forward decidedly in the design and implementation of pro-equity social and health policies at both the local and global levels, this special report reviews the conceptual and methodological framework for tackling health equity. Concepts and methodology are explicitly linked in a practical proposal that promotes the analytical use of subnationally disaggregated administrative data to inform decision-making in that area. This report concludes by proposing the need to institutionalize the measurement, analysis, and monitoring of social disparities in health to create effective national capacity to act on the social and environmental determinants of health and ensure accountability in the commitment to "leave no one behind" on the road to sustainable development, universal health, and social justice.

Health equity; health status disparities; social determinants of health; social theory; epidemiologic measurements.
RESUMO

\section{Da retórica à ação: mensurar as desigualdades em saúde para não deixar ninguém atrás}

Palavras-chave
A equidade em saúde é um princípio norteador da ação em saúde pública cujo propósito nobre é edificar sociedades mais saudáveis e sustentáveis e, ao mesmo tempo, mais justas e inclusivas. Isso está refletido no compromisso mundial de "não deixar ninguém atrás" que guia a Agenda 2030 para o Desenvolvimento Sustentável, apesar de nenhuma das 169 metas estabelecer de forma conceitual ou quantitativa a redução das desigualdades em saúde. Reconhecendo a urgência de transcender a retórica e avançar na formulação e implementação de políticas sociais e de saúde pró-equitativas do nível local ao global, são revistas as bases conceituais e metodológicas para a abordagem da equidade em saúde, vinculadas explicitamente em uma proposta instrumental e prática que promove o uso analítico dos dados administrativos disponíveis desagregados ao nível subnacional para subsidiar a tomada de decisão. Em conclusão, faz-se necessário institucionalizar a mensuração, análise e monitoramento das desigualdades sociais em saúde para efetivamente estabelecer capacidades nacionais para atuar nos determinantes sociais e ambientais da saúde e prestar contas quanto ao compromisso de não deixar ninguém atrás no rumo ao desenvolvimento sustentável, saúde universal e justiça social.

Equidade em saúde; disparidades nos níveis de saúde; determinantes sociais da saúde; teoria social; medidas em epidemiologia. 